\title{
Electric Interaction between Two Identical Conducting Spheres in a Uniform Electric Field
}

\author{
X. GAO ${ }^{a, *}$, Q. WANG ${ }^{b}$, C.-X. $\mathrm{LI}^{b}, \mathrm{~L} \mathrm{HU}^{a}$ AND G. $\mathrm{SUN}^{b}$ \\ ${ }^{a}$ College of Science, Guizhou University, Guiyang 550025, China \\ ${ }^{b}$ Beijing National Laboratory for Condensed Matter Physics and Key Laboratory of Soft Matter Physics, \\ Institute of Physics, Chinese Academy of Sciences, Beijing 100190, China
}

(Received January 18, 2015; in final form October 2, 2015)

\begin{abstract}
An experimental apparatus is devised to measure the interaction between two spheres separated by a small gap in a uniform electric field. The results show that the interaction between two conducting spheres is near that between two dielectric spheres with high permittivity. Accordingly, the calculation can be simplified by mirror image method, for it is only available for conducting system. A method using multiple mirror images of point charges is put forward to analyze the induction of two identical conducting spheres in a uniform electric field. The key operation on how to add compensative charges is emphasized and given out in detail. The results from experiment and calculation are compared, and they agree with each other very well.
\end{abstract}

DOI: $10.12693 /$ APhysPolA.128.289

PACS: $41.20 . \mathrm{Cv}, 47.65 . \mathrm{Gx}$

\section{Introduction}

Interaction between spheres in electric fields is an important issue which is the most basic problem in broad fields such as electrorheological fluids [1-4], electrophoresis $[5,6]$, and other fields [7-9]. Some works from experiments, theory and calculation on two spheres in electric fields have been done [10-16]. Wang $[9,10,12]$ and Tao [15] have carried experiments respectively to show the direct results of interaction between two dielectric spheres, and part of the experimental results do not agree with the theoretical results $[17,18]$. There are many theoretical models to deal with this system with two spheres, such as finite elements method [14], dipole approximation [19], multipole-expansion theory [20-24] and so on. The experimental works show that the measured force is much higher than that calculated by dipole approximation. Furthermore, even calculating by improved dipole model, there are still distinct divergences between the results from experiment and calculation. Cox et al. have developed a calculation method which includes the higher order of polarization, and the result agrees well with the experimental result [22]. Thereafter, a multiple scattering method has been adopted by Jiao et al. which also includes higher order of polarization by a numerical algorithm and can be widely used in almost all kinds of system composed of spheres [24]. When the gap between two spheres is much less than the radius of spheres, the methods adopted by both Cox and Jiao need to spend vast time to give out the result because the very high orders of polarization should be concerned. However, if the spheres are ideal conductors, method of mirror images will be a powerful tool to analyze the electric induction.

* corresponding author; e-mail: gaoxin0526@163.com
Method of images is an old way to deal with simple electrostatic problem in many symmetrical systems which is first developed by Thomson [25]. This method gives simple and direct physical picture which is easy to be accepted, and the results of electric field and potential by this method are analytic solutions. Therefore, it is used to deal with many problems involving conductor systems [26-28]. It is also applied widely to the problems with two conducting spheres [29-34]. In our previous work, a new skill has been put forward to advance the method of multiple image, which need only to calculate point charge images instead of multiple moments [35].

Though some analysis and calculation on interaction between two conducting spheres in electric field have been done before, the former works all focus on the theoretical discussion. There are still little cases to calculate an actual experiment system with specific parameters, and there is no case to finish both calculation and experiment in one work to compare the results yet. Thereby an experiment is devised to measure the electric interaction between two identical conducting spheres. The forces for different magnitude of electric field and different gap between two spheres can be measured. The results obtained from both experiment and calculation can be compared as well.

In Sect. 2 the experimental apparatus is introduced, and the experimental results are given out by figures. The method of multiple images is shown in detail in Sect. 3. In Sect. 4, the calculation for the system used in experiment has been done. The results from both experiment and calculation are compared and discussed. At last, some views for this work are concluded in Sect. 5.

\section{Experiment and results}

The apparatus used in this experiment is shown in Fig. 1, which consists of two horizontal fixed copper plates $(160.0 \mathrm{~mm}$ in diameter) separated by a distance 
of $25.0 \mathrm{~mm}$ by Teflon poles, a stepper motor controlled by a computer, two titanium spheres with diameter of $6.3 \mathrm{~mm}$, an electronic balance with precision of $1.0 \mathrm{mg}$ and a grating ruler connected to a computer with precision of $0.1 \mu \mathrm{m}$. The lower sphere is fixed on the lower electrode plate by an insulating alumina tube with thin diameter of $1.2 \mathrm{~mm}$, while the upper one is stuck to another alumina tube and is hung to the hook under the balance through a small aperture in the center of upper electrode plane. Firstly the connecting line of two spheres' centers is adjusted to be vertical by four micrometers in different directions, and the stepper motor lifts to make the two spheres touch exactly. In this way, the gap between spheres is just the displacement of the stepper motor when it lowers with the control of a computer. The force applied to the upper sphere can be given out by the balance after different voltage is applied to the two electrode planes. Here we adopt AC voltage which frequency is fixed to $50 \mathrm{~Hz}$, because the interaction in electrostatic field is unexpected in practice. We will give out our result on electrostatic condition in future work. Here, the magnitude of the electric field indicates the effective value.

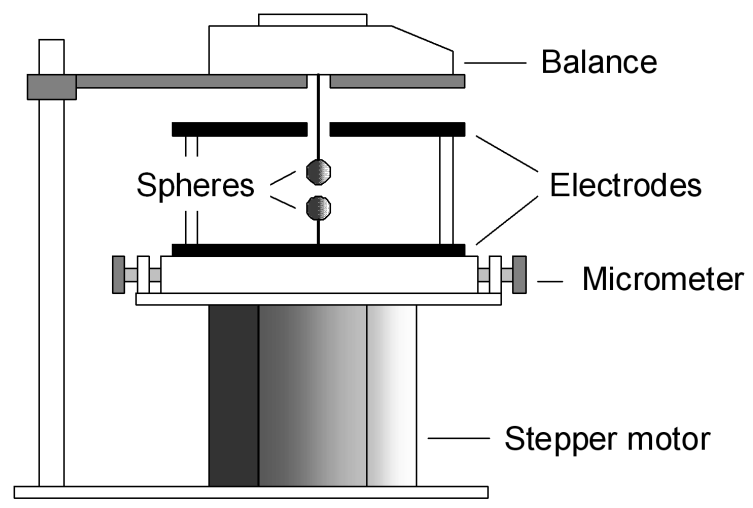

Fig. 1. Sketch of the apparatus used in the experiment.

First, the forces between both conducting spheres and dielectric spheres of $\mathrm{BaTiO}_{3}$ are measured to illustrate that the force induced by applied electric fields between conducting spheres are nearly equal to that between dielectric spheres with high permittivity. The material of $\mathrm{BaTiO}_{3}$ used in this work is polycrystal ceramics whose permittivity is about 3000 at room temperature [12]. The forces between conducting spheres and between $\mathrm{BaTiO}_{3}$ spheres are measured for the cases where gaps between two spheres are 10, 50, and $100 \mu \mathrm{m}$, respectively, and the results are compared as shown in Fig. 2. It can be found that the forces between conducting spheres and between $\mathrm{BaTiO}_{3}$ spheres satisfy the same laws and are really near equality no matter how small the gap is.

The result of experiment in Fig. 3 shows the relation of force and the magnitude of applied electric field for different gap $\delta$. The forces should satisfy quadratic power law with $\boldsymbol{E}_{0}$ theoretically [35], so the fitting of parabola is adopted and the fitting curves are shown in Fig. 3, too. By the results of fitting, it can be found that the

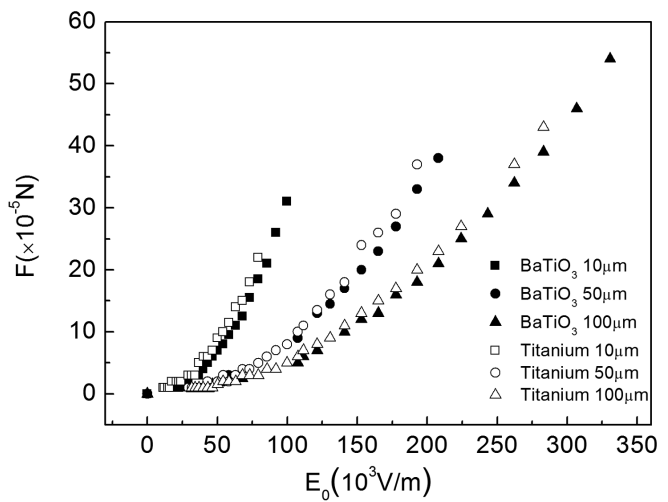

Fig. 2. Comparision of interactions between two dielectric spheres with high permittivity and between two conducting spheres.

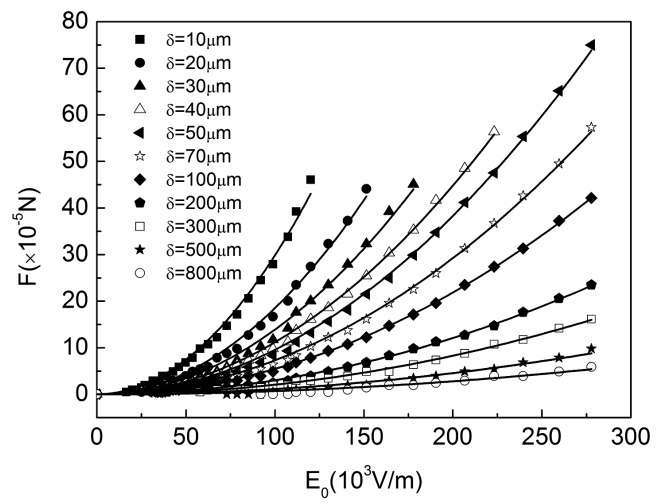

Fig. 3. Forces versus applied electric fields $E_{0}$ for a series of gap $\delta$. The symbols are for the measured data and the curves are fitting quadratic function from the data.

force satisfies quadratic power law well. When the gap size changes to smaller value, the force increases faster with the electric field applied. This means that the force will be more and more intense for smaller gap size when applied electric field is fixed to a constant.

\section{Calculation}

When a point charge or a dipole moment are out of a conducting sphere, the electric field out of sphere can be taken as one produced by both the charge or dipole moment and their images in the sphere [33, 34, 36, 37]. For point charge $Q_{0}$, its mirror image charge in the sphere with radius of $R$ :

$$
Q=-\frac{R}{d} Q_{0},
$$

and the position

$$
r=\frac{R^{2}}{d}
$$

where $d$ is the distance between the point charge and the center of the sphere, $r$ is the distance between the image charge $Q$ and the center of the sphere. To keep the sphere neutral, another compensative point charge $Q^{\prime}$ at the centre of the sphere is needed 


$$
Q^{\prime}=-Q
$$

For dipole moment $\boldsymbol{p}_{0}$, its images conclude a dipole moment $\boldsymbol{p}$, a point charge $q$ and compensative point charge $q^{\prime}$ at the center of the sphere

$$
\begin{aligned}
& \boldsymbol{p}=\left(\frac{R}{d}\right)^{3} \boldsymbol{p}_{0}, \\
& q=-\frac{R}{d^{2}} p, \\
& q^{\prime}=-q=\frac{R}{d^{2}} p .
\end{aligned}
$$

The positions of $\boldsymbol{p}$ and $q$ are same as Eq. (2), and the position of $q^{\prime}$ is the same as $Q^{\prime}$.

When a conducting sphere with radius of $R$ is positioned in an applied uniform electrostatic field, charge distribution is induced, and electric field near the surface of sphere is not uniform any more. In this condition, electric field out of the spheres can be thought as one produced by a dipole moment in the center of sphere instead of the charge distribution in the surface. When an electric field $\boldsymbol{E}_{0}$ is applied, the dipole moment can be written as [37-40]:

$$
\boldsymbol{p}_{0}=4 \pi \varepsilon R^{3} \boldsymbol{E}_{0},
$$

where $\varepsilon$ is the permittivity of medium around.

When two spheres approach together, the charge distribution will be distorted by each other. The distance between the centers of spheres is given as

$$
d=2 R+\delta,
$$

where $\delta$ is the gap as shown in Fig. 4. We sign the left sphere as B, right sphere as A, and the direction of uniform electrostatic field $\boldsymbol{E}_{0}$ is parallel to the connecting line of two spheres' centers whose unit direction vector is signed as $\widehat{\boldsymbol{E}}_{0}$.

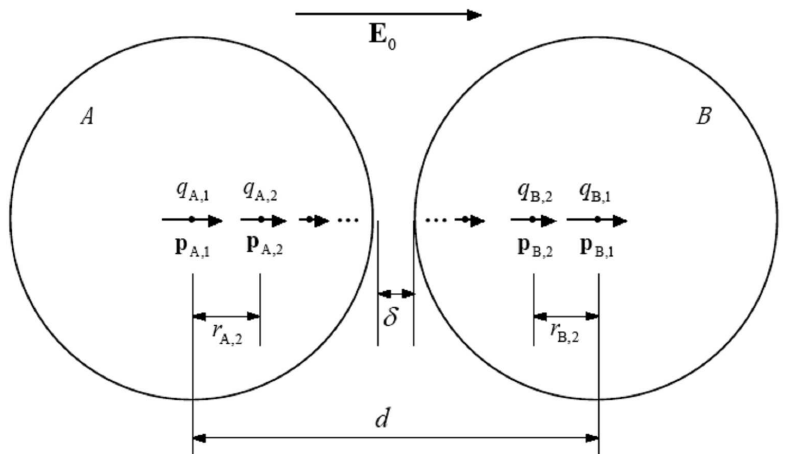

Fig. 4. Sketch map of image charges distribution in conducting spheres.

Firstly, the first order images are induced by only uniform fields, which are signed as

$$
\boldsymbol{p}_{\mathrm{A}, 1}=\boldsymbol{p}_{\mathrm{B}, 1}=4 \pi \varepsilon_{0} R^{3} \boldsymbol{E}_{0} \text {. }
$$

Because the two spheres are both not charged, in order to keep both spheres neutral, the first order compensative charges in the centers of two spheres

$$
q_{\mathrm{A}, 1}=q_{\mathrm{B}, 1}=0 .
$$

The positions of the compensative charges are signed as the distance to the centers

$$
r_{\mathrm{A}, 1}=r_{\mathrm{B}, 1}=0 \text {. }
$$

As Eqs. (4), (5) and (6), the first order images in sphere B will produce second order images in sphere A:

$$
\begin{aligned}
& \boldsymbol{p}_{\mathrm{A}, 2}=\left(\frac{R}{d}\right)^{3} \boldsymbol{p}_{\mathrm{B}, 1}=\left(\frac{R}{d}\right)^{3} \boldsymbol{p}_{\mathrm{A}, 1}, \\
& q_{\mathrm{A}, 2}=-\frac{R}{d^{2}} p_{\mathrm{B}, 1}=-\frac{R}{d^{2}} p_{\mathrm{A}, 1},
\end{aligned}
$$

whose position is signed by the distance to center of $\mathrm{A}$ in the connecting line between two spheres' centers as

$$
r_{\mathrm{A}, 2}=\frac{R^{2}}{d} .
$$

Similarly, the second images in sphere B can be given as

$$
\begin{aligned}
& \boldsymbol{p}_{\mathrm{B}, 2}=\left(\frac{R}{d}\right)^{3} \boldsymbol{p}_{\mathrm{A}, 1}, \\
& q_{\mathrm{B}, 2}=\frac{R}{d^{2}} p_{\mathrm{A}, 1},
\end{aligned}
$$

and the position is also signed as

$$
r_{\mathrm{B}, 2}=\frac{R^{2}}{d} .
$$

To keep the two spheres neutral, compensative charges is needed in the centers of two spheres

$$
\begin{aligned}
& q_{\mathrm{A}, 1}=-q_{\mathrm{A}, 2}=\frac{R}{d^{2}} p_{\mathrm{A}, 1}, \\
& q_{\mathrm{B}, 1}=-q_{\mathrm{B}, 2}=-\frac{R}{d^{2}} p_{\mathrm{B}, 1} .
\end{aligned}
$$

Here it should be mentioned that $q_{\mathrm{A}, 1}, q_{\mathrm{B}, 1}$ are always the compensative charges in spheres' centers, and they will produce image charges at higher order images calculation as well. Furthermore, because the new compensative charges emerge in the next iterative steps, the value of $q_{\mathrm{A}, 1}, q_{\mathrm{B}, 1}$ will change at every step.

Following the same algorithm, the higher order images in sphere $\mathrm{A}$ and their positions can be given as

$$
\begin{aligned}
& \boldsymbol{p}_{\mathrm{A}, i}=\left(\frac{R}{d-r_{\mathrm{B}, i-1}}\right)^{3} \boldsymbol{p}_{\mathrm{B}, i-1}= \\
& \left(\frac{R}{d-r_{\mathrm{A}, i-1}}\right)^{3} \boldsymbol{p}_{\mathrm{A}, i-1}, \\
& q_{\mathrm{A}, i}=\frac{R}{\left(d-r_{\mathrm{B}, i-1}\right)^{2}} p_{\mathrm{B}, i-1}-\frac{R}{d-r_{\mathrm{B}, i-1}} q_{\mathrm{B}, i-1}= \\
& \quad \frac{R}{\left(d-r_{\mathrm{B}, i-1}\right)^{2}} p_{\mathrm{A}, i-1}+\frac{R}{d-r_{\mathrm{A}, i-1}} q_{\mathrm{A}, i-1}, \\
& r_{\mathrm{A}, i}=\frac{R^{2}}{d-r_{\mathrm{B}, i-1}}=\frac{R^{2}}{d-r_{\mathrm{A}, i-1}} .
\end{aligned}
$$

Similarly, those in sphere B can be described as

$$
\boldsymbol{p}_{\mathrm{B}, i}=\left(\frac{R}{d-r_{\mathrm{A}, i-1}}\right)^{3} \boldsymbol{p}_{\mathrm{B}, i-1},
$$




$$
\begin{aligned}
q_{\mathrm{B}, i} & =-\frac{R}{\left(d-r_{\mathrm{B}, i-1}\right)^{2}} p_{\mathrm{B}, i-1}+\frac{R}{d-r_{\mathrm{B}, i-1}} q_{\mathrm{B}, i-1}, \\
r_{\mathrm{B}, i} & =\frac{R^{2}}{d-r_{\mathrm{B}, i-1}} .
\end{aligned}
$$

Here $i$ indicates the number of iterative step, and $q_{\mathrm{B}, i}$ includes the images of $p_{\mathrm{B}, i-1}$ and $q_{\mathrm{B}, i-1}$. It should be noted that all $q_{\mathrm{A}, j}, q_{\mathrm{B}, j}(j=2,3, \ldots, i)$ should be calculated again after the calculation for $i$-order images is finished, and the compensative charges in centers are calculated as

$$
\begin{aligned}
& q_{\mathrm{A}, 1}=-\sum_{j=2}^{i} q_{\mathrm{A}, j}, \\
& q_{\mathrm{B}, 1}=-\sum_{j=2}^{i} q_{\mathrm{B}, j} .
\end{aligned}
$$

Once the series of images are given by calculation, the force acted on sphere B induced by both applied electric field $\boldsymbol{E}_{0}$ and charge distribution on sphere A can be calculated as

$$
\begin{gathered}
\boldsymbol{F}_{\mathrm{B}}=\sum_{i} \sum_{j}\left(\frac{q_{\mathrm{B}, i} q_{\mathrm{A}, j}}{4 \pi \varepsilon_{0}\left(d-r_{\mathrm{B}, i}-r_{\mathrm{A}, j}\right)^{2}}\right. \\
\left.+\frac{q_{\mathrm{B}, i} p_{\mathrm{A}, j}}{2 \pi \varepsilon_{0}\left(d-r_{\mathrm{B}, i}-r_{\mathrm{A}, j}\right)^{3}}\right) \widehat{\boldsymbol{E}}_{0} \\
+\sum_{i} \sum_{j}\left(\frac{q_{\mathrm{A}, i} p_{\mathrm{B}, j}}{2 \pi \varepsilon_{0}\left(d-r_{\mathrm{B}, i}-r_{\mathrm{A}, j}\right)^{3}}\right. \\
\left.-\frac{p_{\mathrm{B}, i} p_{\mathrm{A}, j}}{4 \pi \varepsilon_{0}\left(d-r_{\mathrm{B}, i}-r_{\mathrm{A}, j}\right)^{4}}\right) \widehat{\boldsymbol{E}}_{0} .
\end{gathered}
$$

\section{Result and discussion}

To compare with experiment, two conducting spheres with identical radius $R$ of $3.15 \mathrm{~mm}$ which is the actual size of sphere used in our experiment are considered in calculation. The magnitude of applied field is $1.0 \times 10^{5} \mathrm{~V} / \mathrm{m}$. For all of the calculation, we set iterative number as 1000 to ensure the perfect precision [35].

The method used here calculates electric force by summation of all interactions between every pair of images in different spheres as Eq. (28). From Eq. (28), it can be known that the force is proportional to $q_{\mathrm{B}, i} q_{\mathrm{A}, j}, q_{\mathrm{B}, i} p_{\mathrm{A}, j}$, $q_{\mathrm{A}, i} p_{\mathrm{B}, j}, p_{\mathrm{B}, i} p_{\mathrm{A}, j}$ and $q_{\mathrm{B}, i} \boldsymbol{E}_{0}$. Based on Eqs. (20), (21), every order images are proportional to the last order images and then to the first order images which are proportional to the magnitude of applied electric field $\boldsymbol{E}_{0}$. Consequently, the calculated force must satisfy quadratic power law in nature, so the forces are normalized by $\left|\boldsymbol{E}_{0}\right|^{2}$ in order to describe features of this system without considering the effect of magnitude of applied electric field. The normalized forces for different gap sizes are calculated by method advanced here as the line shown in Fig. 5.

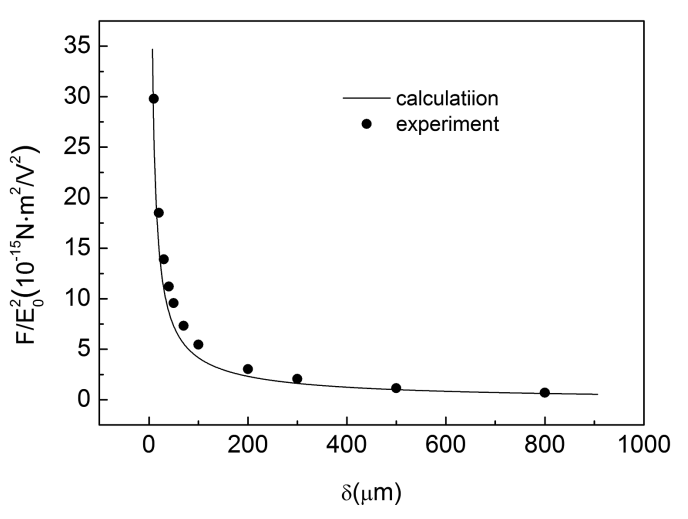

Fig. 5. Comparison of the ratio of $F / E_{0}^{2}$ from calculation with that from experiment.

To compare the results from experiment and calculation clearly, the forces normalized by $\left|\boldsymbol{E}_{0}\right|^{2}$ for different gap sizes measured by experiment are shown by circle symbols in Fig. 5. Here we must mention that the data of $F / E_{0}^{2}$ from experiment are given by fitting of parabola from direct experimental data. From Fig. 5, it can be found that the results from both experiment and calculation agree with each other in an acceptable accuracy. The results in Fig. 5 also show that the force will increase much faster with increase of $\boldsymbol{E}_{0}$ when two spheres approach with smaller gap. This means that the interaction induced by applied electric field will be enhanced remarkably when gap size gets smaller.

\section{Conclusion}

An experiment is devised to measure interaction force of two conducting spheres and two dielectric spheres with high permittivity in an applied electric field. The changing laws of electric forces between two spheres with different gap size and different magnitude of applied electric field have been presented by experimental measurement. Through comparing the forces between two conducting spheres and between two $\mathrm{BaTiO}_{3}$ spheres, it can be conclude that the force between two conducting spheres is similar and nearly equal to that between two dielectric spheres with high permittivity. This means that we can learn features of polarization and interaction of dielectrics by studying system composed with conductors, because calculation for the conductors in applied electric fields is convenient and accurate based on image method.

A method of multiple images is adopted and advanced to analyze induction of two identical conducting spheres in a uniform electrostatic field. When two conducting spheres approach each other in a uniform electric field, a series of image charges and dipoles are calculated by iteration instead of calculating the distribution of charges on the surface of conducting spheres. Once the image charges and dipoles are determined, we can get electric force acting on conducting spheres by calculating all interactions between image charges, and this method can save much time in process of calculation. 
Both results from experiment and calculation show that the electric force applied on each sphere satisfies the quadratic power law. For comparing, both forces calculated and measured are normalized by $\left|\boldsymbol{E}_{0}\right|^{2}$. The relation between normalized forces and gap size $\delta$ is given out also, and the relation shows that the force will be much more intense when $\delta$ gets smaller. The most importance is that the normalized forces from experiment and calculation agree with each other very well in high accuracy. The agreement proves that the experimental device is viable to measure electric force and method of multiple mirror images is a powerful means to calculate electric force.

The analysis may be extended to deal with system with multiple conducting spheres. This method also provides a possible way to analyze the electrorheological fluid with metal particles [41, 42], and interaction of dispersed dielectric particles with surface conductance or high permittivity in colloid system can be calculated as well.

\section{Acknowledgments}

This work was supported by National Natural Science Foundation of China (11304049, 11264006), Guizhou Provincial Science and Technology Foundation (J[2010]2132) and Doctor Funds of Guizhou University ([2012]020).

\section{References}

[1] R. Tao, Q. Jiang, H.K. Sim, Phys. Rev. E 52, 2727 (1995).

[2] W.J. Tian, M.K. Liu, J.P. Huang, Phys. Rev. E $\mathbf{7 5}$, 021401 (2007).

[3] K. Kim, D. Stroud, X. Li, D.J. Bergman, Phys. Rev. E 71, 031503 (2005).

[4] H. Ma, W. Wen, W.Y. Tam, P. Sheng, Phys. Rev. Lett. 77, 2499 (1996).

[5] Z. Qiu, N. Markarian, B. Khusid, A. Acrivos, J. Appl. Phys. 92, 2829 (2002).

[6] H.J. Keh, S.B. Chen, J. Colloid Interface Sci. 130, 542 (1989).

[7] W. Chen, S. Tan, T.K. Ng, W.T. Ford, P. Tong, Phys. Rev. Lett. 95, 218301 (2005).

[8] J.H. Cloete, J. van der Merwe, IEEE Trans. Educ. 41, 141 (1998).

[9] Z. Wang, Z. Peng, K. Lu, Appl. Phys. Lett. 82, 1796 (2003).

[10] Z. Wang, R. Shen, X. Niu, K. Lu, J. Appl. Phys. 94, 7832 (2003).

[11] J.C. Crocker, D.G. Grier, Phys. Rev. Lett. 73, 352 (1994).

[12] Z. Wang, R. Shen, X. Niu, G. Sun, K. Lu, B. Hou, W. Wen, J. Phys. D Appl. Phys. 38, 1325 (2005).

[13] L. Gao, J.T.K. Wan, K.W. Yu, Z.Y. Li, Phys. Rev. E 61, 6011 (2000).

[14] R. Tao, J.M. Sun, Phys. Rev. Lett. 67, 398 (1991).

[15] R. Tao, Y.C. Lan, Phys. Rev. E. 72, 041508 (2005).

[16] E. Bichoutskaia, A.L. Boatwright, A. Khachatourian, A.J. Stace, J. Chem. Phys. 133, 024105 (2010).
[17] W. Wen, H. Ma, W. Tam, P. Shen, Phys. Rev. E 55, R1294 (1997).

[18] T. Nagaya, N. Fujimoto, T. Miki, H. Orihara, Y. Ishibashi, M. Doi, J. Phys. Soc. Japan 63, 2581 (1994).

[19] D.J. Klingenberg, F. van Swol, C.F. Zukoski, J. Chem. Phys. 94, 6160 (1991).

[20] H.J.H. Clercx, G. Bossis, Phys. Rev. E 48, 2721 (1993).

[21] H.J.H. Clercx, G. Bossis, J. Chem. Phys. 103, 9426 (1995).

[22] B.J. Cox, N. Thamwattana, J.M. Hill, Appl. Phys. Lett. 88, 152903 (2006).

[23] Y. Chen, A.F. Sprecher, H. Conrad, J. Appl. Phys. 70, 6796 (1991).

[24] M.C. Jiao, G. Sun, Q. Wang, K.Q. Lu, Mod. Phys. Lett. B 26, 115007 (2012).

[25] W. Thomson, Reprint of Papers on Electrostatics and Magnetism, 2nd ed., MacMillan, London 1884

[26] W.E. Smith, J. Rungis, J. Phys. E Sci. Instrum. 8, 379 (1975).

[27] J.C.-E. Sten, K.I. Nikoskinen, J. Electrostat. 35, 267 (1995).

[28] B. Techaumnata, T. Takuma, J. Electrostat. 64, 165 (2006).

[29] L. Poladian, Q. J. Mech. Appl. Math. 41, 395 (1988).

[30] D.J. Jeffrey, Y. Onishi, J. Phys. A 13, 2847 (1980).

[31] A. Moussiaux, A. Ronveaux, J. Phys. A 12, 423 (1979).

[32] J. Slisko, R.A. Brito-Orta, Am. J. Phys. 66, 352 (1998).

[33] B.U. Felderhof, D. Palaniappan, J. Appl. Phys. 86, 6501 (1999).

[34] G. Tong, Eur. J. Phys. 17, 244 (1996).

[35] X. Gao, L. Hu, G. Sun, Commun. Theor. Phys. 57, 1066 (2012).

[36] H.F.M. van den Bosch, K.J. Ptasinski, P.J.A.M. Kerkhof, J. Appl. Phys. 78, 6345 (1995).

[37] T.B. Jones, Electromechanics of Particles, Cambridge University Press, Cambridge 1995.

[38] J.A. Stratton, Electromagnetic Theory, McGraw-Hill, New York 1941

[39] Z. Jiang, J. Electrostat. 58, 247 (2003).

[40] Z. Jiang, Z. Shen, K. Lu, J. Electrostat. 53, 53 (2001).

[41] W. Wen, K. Lu, Appl. Phys. Lett. 67, 2147 (1995).

[42] W. Wen, K. Lu, Phys. Fluids 8, 2789 (1996). 